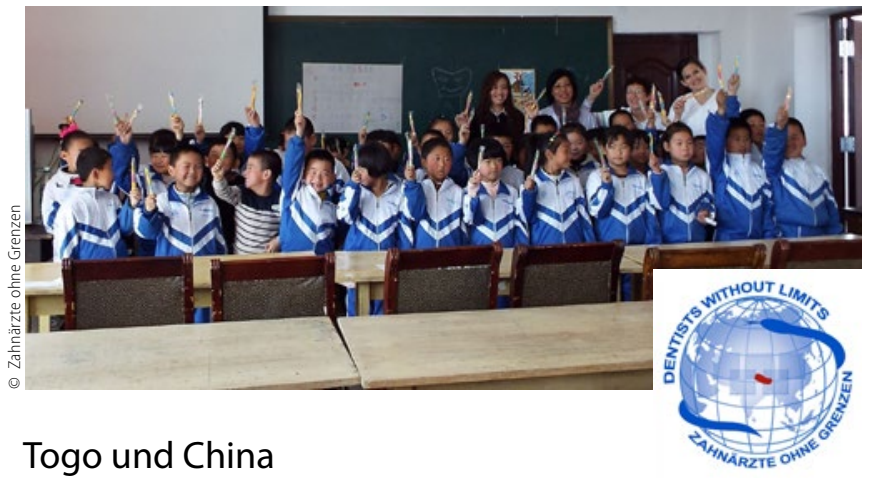

\section{Neue Hilfseinsätze der Stiftung Zahnärzte ohne Grenzen}

Die Stiftung Zahnärzte ohne Grenzen setzt auf weitere ehrenamtliche Unterstützung durch Zahnärzte in diversen Einsatzgebieten im Ausland. Für interessierte Einsatzteilnehmer bietet sich mit einem Engagement in Togo ein weiteres karitatives Betätigungsfeld. Nach einem ersten Einsatz im Frühjahr geht die zahnärztliche Hilfe auch in China weiter. Seit September starten jeden Monat gemischte Teams der Dentists without Limits Foundation (DWLF), wie die internationale Stiftung heißt, in die Hilfsgebiete, um dort die zahnmedizinische Versorgung der Bevölkerung zu verbessern und Standards für eine Basiszahnmedizin einzuführen. In Togo arbeiten die Vierergruppen von Aného aus mit mobilen Zahnkliniken, die es vor Ort bereits gibt, und werden von einem einheimischen Zahnarzt bei der Arbeit begleitet. In China arbeiten Zahnärzte von Anshan aus. Einheimische Helfer und Dolmetscher begleiten die Arbeit. Interessenten für einen Hilfseinsatz können sich per Mail unter info@ dwlf.org mit der Stiftung in Verbindung setzen. Die Stiftung wurde 2004 von dem Nürnberger Kieferorthopäden Dr. Claus Macher gegründet. Zahlreiche Zahnärzte waren seither in vielen Ländern ehrenamtlich im Einsatz.

sas
KBV-Versichertenbefragung

\section{Patienten haben hohes Vertrauen in den Arzt}

Patienten haben niedergelassenen Ärzten und Psychotherapeuten ein gutes Zeugnis ausgestellt. Nachholbedarf gibt es in den Praxen allerdings bei der Vertraulichkeit am Empfang. Zu diesem Ergebnis kommt die Forschungsgruppe Wahlen, die im Auftrag der Kassenärztlichen Bundesvereinigung (KBV) etwa 6100 Versicherte befragt hat. Demnach bewerten mehr als 90 Prozent der Befragten das Vertrauensverhältnis zu ihrem Arzt als „gut" bis „sehr gut“. Weitere Spitzenwerte verzeichnete die Ärzteschaft in Sachen Freundlichkeit (96 Prozent), Fachkompetenz (93 Prozent) und Verständlichkeit der Erklärung (92 Prozent). Das politisch viel diskutierte Thema der Wartezeiten auf einen Arzttermin ist nur für eine geringe Zahl der Befragten ein Problem. Lediglich einer von zehn Befragten bewertete die Wartezeit als zu lang. Eine Schwachstelle deckte die Erhebung bei der Frage nach der Vertraulichkeit am Empfang in der Praxis auf. Von den befragten Versicherten schätzten 45 Prozent die Diskretion am Empfang als „weniger gut“ oder „überhaupt nicht gut" ein. Mit Blick auf die Ergebnisse erklärte die stellvertretende KBV-Vorstandsvorsitzende Regina Feldmann: „Wir sollten uns deshalb alle stärker ins Bewusstsein rücken, dass Sensibilität im Umgang mit den Daten unserer Patienten eine wichtige Größe ist, auf die wir besonders achten müssen.“

cas

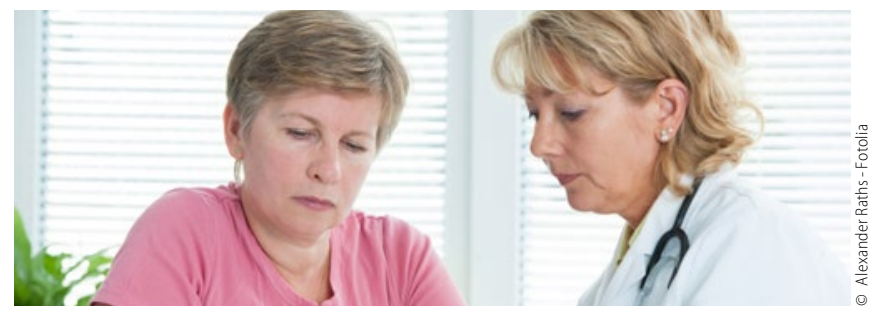

\title{
UPD-Zwischenbilanz
}

\section{Mängel bei Erreichbarkeit und Qualität}

Das privatwirtschaftliche Unternehmen Sanvartis hat seit Jahresbeginn die Trägerschaft für die Unabhängige Patientenberatung Deutschland (UPD) übernommen. Nun hat die Bundesregierung erstmals Zwischenbilanz zur Arbeit des neuen Betreibers gezogen. Dabei spricht die Regierung von technischen Problemen im ersten Halbjahr 2016. Diese seien aber in einem Übergangszeitraum von sechs Monaten eingeplant gewesen.

Bis Ende 2015 war die Angebotsgemeinschaft aus Sozialverband Vdek, Verbraucherzentrale Bundesverband und dem Verbund unabhängige Patientenberatung mit der UPD betraut. Nach einem europaweiten Ausschreibungsverfahren hatte der Duisburger Gesundheitsdienstleister Sanvartis den Zuschlag erhalten. Die Neuvergabe sorgte für scharfe Kritik von Ärzten und Zahnärzten, weil Sanvartis bislang überwiegend als Dienstleister für Krankenkassen und Pharmaindustrie tätig war.

In einer Antwort auf eine Kleine Anfrage der Bundestagsfraktion von Bündnis 90/Die Grünen nannte die Bundesregierung nun kürzlich 15 Beschwerden, die bei dem Patientenbeauftragten bis Mitte dieses Jahres über die Erreichbarkeit und Qualität der UPD eingegangen sind. Nach Angaben der Regierung sind die Beschwerden auf technische Probleme in der Aufbauphase zurückzuführen. Aufgrund des eingeräumten Übergangszeitraums sei ein umfassendes UPD-Beratungsangebot im ersten Halbjahr auch nicht zu erwarten gewesen.

Ein erklärtes Ziel des neuen UPD-Konzeptes waren mehr Patientenkontakte. Aus der aktuellen Antwort der Regierung geht hervor, dass die neue UPD trotz erhöhter Fördermittel Mitte dieses Jahres weniger Kontakte verzeichnen konnte als die „alte“ UPD im Vorjahr. Während die frühere UPD im Juli 2015 etwa 7500 Beratungen durchführte, waren es Mitte dieses Jahres 7000 Kontakte. Die Fördermittel beliefen sich im vergangenen Jahr auf rund 7.060.000 Euro. Für das laufende Jahr stieg die Fördersumme um 27,5 Prozent und beträgt neun Millionen Euro. cas 